\title{
Ziv-Zakai Bound and Multicorrelator Compression for a Galileo E1 Meta-Signal
}

\author{
Carolin Schwalm*, Christoph Enneking**, Steffen Thoelert** \\ ${ }^{*}$ Chair of Navigation, RWTH Aachen University \\ Aachen, GERMANY \\ email: carolin.meyer@rwth-aachen.de \\ postal address: Mies-van-der-Rohe-Str. 15, 52074 Aachen, Germany \\ ${ }^{* *}$ Institute of Communications and Navigation, German Aerospace Center (DLR) \\ Wessling, GERMANY \\ email: \{christoph.enneking, steffen.thoelert\}@dlr.de \\ postal address: Muenchner Str. 20, 82234 Wessling, Germany
}

Associated ENC 2020 topic: System Evolution

We discuss robustness and accuracy of time of arrival (ToA) estimation using meta-signal combining (MSC). MSC is a receive strategy to process multiple navigation signals as a single wideband signal, even though they are transmitted on different carrier frequencies. This strategy was originally proposed due its potentially high ToA estimation accuracy in combination with good spectral efficiency and power efficiency. Furthermore, it offers the possibility to make optimal use of legacy signals and newly added signal components; in this work, we focus on the example of the E1 open serivce (OS) and the forthcoming E1-D signal. We discuss estimation robustness against noise on the basis of the Ziv-Zakai Lower Bound (ZZLB); our analytical results reveal that without large carrier-to-noise density ratio or long observation times, autocorrelation sidelobes make it impossible for an unbiased ToA estimator to attain the excellent performance promised by the simpler Cramér-Rao Lower Bound (CRLB). Furthermore, we demonstrate that a carefully configured bank of correlators should be used in order to avoid further performance losses.

\section{Introduction}

As the services of global navigation satellite systems (GNSS) are constantly being modernized, they provide a variety of legacy signals as well as newly added signals. A recently discussed option in future Galileo signal evolution is the extension of the E1 open service (OS) by introducing a new signal E1-D, located somewhere between 1 to $25 \mathrm{MHz}$ above the E1 OS carrier at $1575 \mathrm{MHz}$. At the receive side, this would offer the possibility to use both the legacy and the 
modernized signal for ranging, exploiting their spectral diversity. One way to achieve this is the concept of meta-signal combining (MSC), which was investigated in [1] and in [2].

Briefly, the idea of MSC consists in processing two (or more) GNSS signals, potentially transmitted at different carrier frequencies, as if they were one single signal. That is, the spectral components from two different bands are processed relative to a virtual center frequency bisecting the combined spectrum, instead of demodulating and processing each component individually at baseband. As a benefit, the bandwidth of the combined signal can be much larger than the bandwidth of the individual components; this leads also to a larger Gabor bandwidth, hence better time of arrival (ToA) estimation performance according to the Cramér-Rao Lower Bound [7].

The robustness of MSC, however, is very poor due to the concentration of the received signal power near the upper and lower end of the receiver bandwidth. Such a power spectral density (PSD) corresponds to an autocorrelation function (ACF) with a sharp main peak that comes along with considerable side peaks. When observed under noise, these side peaks can rise above the main peak and produce large estimation errors. Due to their lack of an unambiguous main peak, meta-signals belong to the class of ambiguity-prone signals [3]. For this class, the CRLB on ToA estimation variance leads to overoptimistic results. While the CRLB is still valid as a lower bound, it cannot serve as a benchmark that an optimal estimator can actually attain: in other words, it is not a tight bound. A tight bound can be given, however, by the Ziv-Zakai Bound (ZZB) [3].

The contribution of this work is twofold. Firstly, we calculate and discuss CRLB and ZZB for an exemplary E1 meta-signal with spectral mainlobes separated by up to $25 \mathrm{MHz}$. We shall observe that not even a maximum likelihood estimator (MLE) with full knowledge of the received signal will be able to attain the CRLB in low-to-medium SNR. This analytical result essentially confirms the findings of the experimental study [1], which showed that MSC is not practicable in dynamic conditions or with low-to-medium carrier-to-noise density ratio $\left(C / N_{0}\right)$. Our second contribution is intended for the high SNR regime, where the ZZB coincides with the CRLB and high accuracy is attainable by MSC. In this regime, the ACF sidelobes are not as hazardous anymore because noise is not able to lift them above the central peak. The receiver must, however, ensure to estimate the correct global maximum of the ACF rather than a local maximum - to this end, a correlator bank rather than the conventional two early/late correlators is necessary [4]. We determine the optimal spacing and number of correlators via a simple estimation theoretic argument: passing the received signal through a bank of correlators is a compression step that comes with an information loss. By comparing the Fisher information measure (the inverse of the CRLB) before and after the compression and requiring that the two are nearly equal, we can determine feasible configurations of the correlator bank. Our results from theory and simulation demonstrate that at least 15 correlators should be employed for robust MSC in the high-SNR regime, if the E1/E1-D carriers are separated by $25 \mathrm{MHz}$. 


\section{Signal Model}

For the discussion, consider a radio frequency power spectrum like the one shown in Fig. 1. It consists of the power spectra of two individual pseudorandom noise (PRN) signals: a binary offset carrier $(\mathrm{BOC}(1,1))$ spectrum at carrier frequency $f_{1}$, and a binary-phase shift keying (BPSK(1)) signal at carrier frequency $f_{2}$. The common chip-time is $T_{c}$. Choosing the virtual carrier frequency $\left(f_{1}+f_{2}\right) / 2$, the baseband power spectrum can be given as

$$
\tilde{\Phi}(f)=C_{1} T_{c}\left(\operatorname{sinc}\left(\left(f+f_{M}\right) T_{c}\right) \tan \left(\pi\left(f+f_{M}\right) T_{c} / 2\right)\right)^{2}+C_{2} T_{c}\left(\operatorname{sinc}\left(\left(f-f_{M}\right) T_{c}\right)\right)^{2},
$$

where $f_{M}=\left(f_{2}-f_{1}\right) / 2$. In the above equation, the first summand represents the BOC component and the second summand represents the BPSK component. The constants $C_{1}$ and $C_{2}$ have dimension Watt and indicate the power of the individual components. We assume that only the signal mainlobes are processed, which are indicated by the shaded blue area in Fig. 1. Therefore, the final PSD of the received baseband signal is given by

$$
\Phi(f)= \begin{cases}C_{1} T_{c}\left(\operatorname{sinc}\left(\left(f+f_{M}\right) T_{c}\right) \tan \left(\pi\left(f+f_{M}\right) T_{c} / 2\right)\right)^{2} & \text { if }\left|f+f_{M}\right| \leq 2 / T_{c} \\ C_{2} T_{c}\left(\operatorname{sinc}\left(\left(f-f_{M}\right) T_{c}\right)\right)^{2} & \text { if }\left|f-f_{M}\right| \leq 1 / T_{c} \\ 0 & \text { else. }\end{cases}
$$

We denote the strictly limited receiver passband $\mathcal{B}=\left[-f_{M}-\frac{2}{T_{c}},-f_{M}+\frac{2}{T_{c}}\right] \cup\left[f_{M}-\frac{1}{T_{c}}, f_{M}+\frac{1}{T_{c}}\right]$. Note also that this is an asymmetric PSD. The corresponding complex-valued ACF

$$
\phi(\tau)=\int_{\mathcal{B}} \Phi(f) e^{\mathrm{j} 2 \pi f \tau} \mathrm{d} \tau
$$

is shown in Fig. 2.

Let $s(t)$ be the noiseless analytic baseband signal with PSD given by (2). It can be given in terms of baseband PRN signals $s_{1}(t)$ and $s_{2}(t)$ as follows

$$
s(t)=\sqrt{C_{1}} e^{j \varphi_{1}} s_{1}(t) e^{-j \pi f_{M} t}+\sqrt{C_{2}} e^{j \varphi_{2}} s_{2}(t) e^{j \pi f_{M} t},
$$

where $\varphi_{1}, \varphi_{2}$ represent (unknown) phase offsets.

\section{Performance Bounds for Time of Arrival Estimation}

We consider the problem of estimating the unknown time of arrival (ToA) $\tau$ based on a noisy observation

$$
y(t)=s(t-\tau)+n(t), \quad t \in\left[0, T_{i}\right]
$$

where the real and imaginary part of $n(t)$ are independent white Gaussian noise processes, each with double-sided PSD $N_{0} / 2$. 


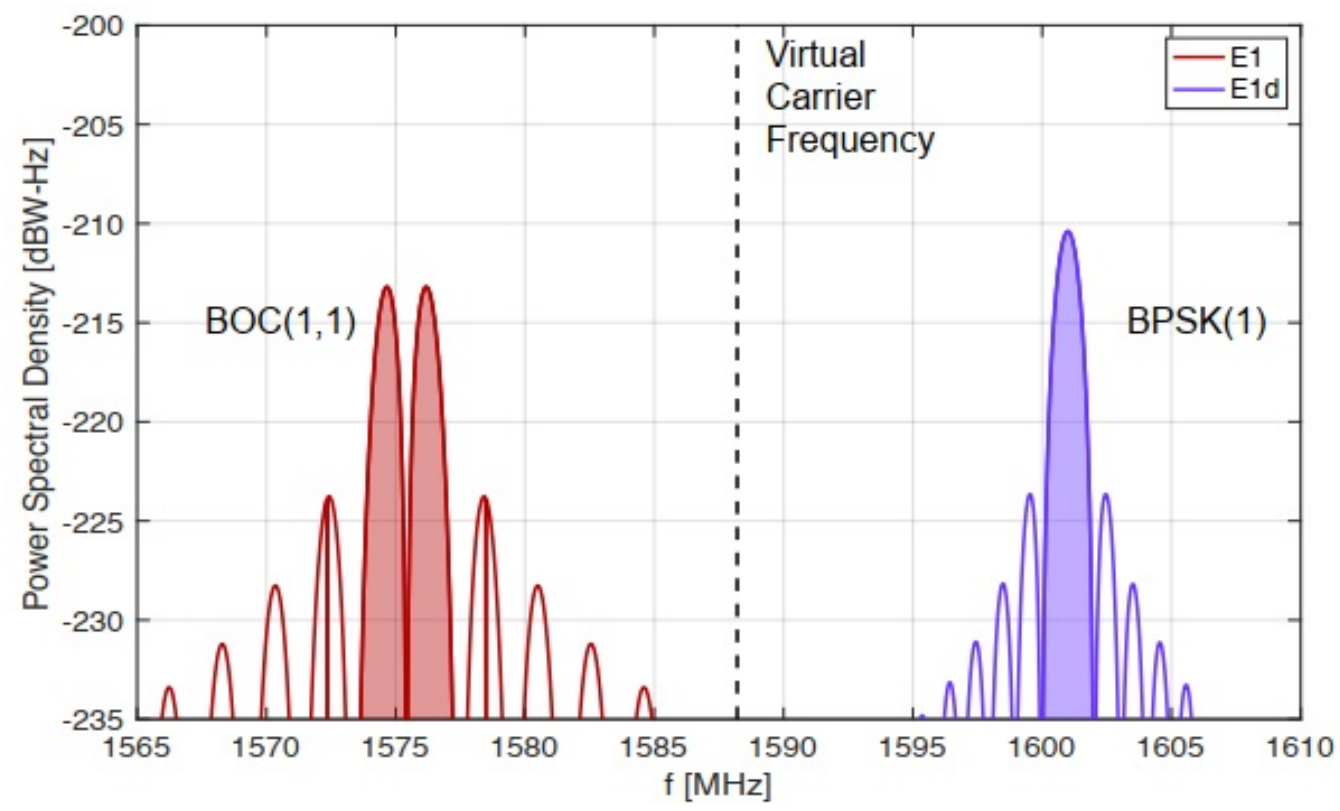

Figure 1: Radio frequency PSD of a BOC signal at $f_{1}=1575.42 \mathrm{MHz}$ and a BPSK signal at $f_{2}=1600.995$. Signal mainlobes are shaded.

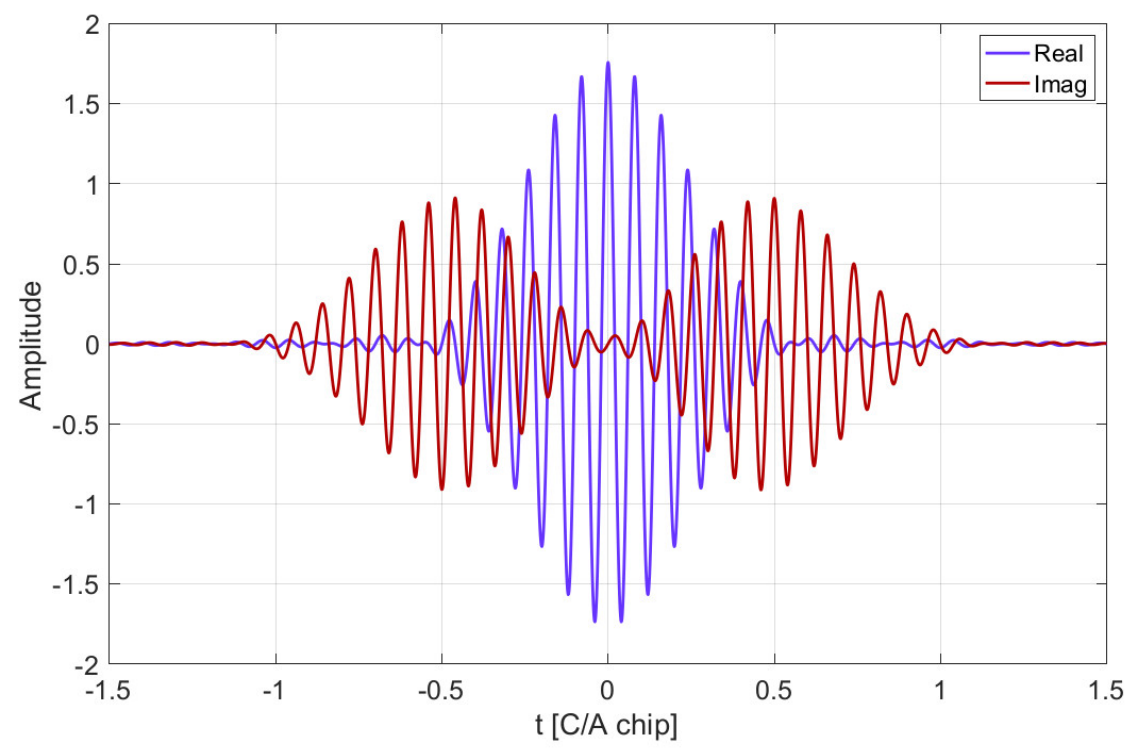

Figure 2: Autocorrelation function of the BOC/BPSK meta-signal. 
Let us define the carrier-to-noise-density ratio of the bandlimited meta-signal

$$
C / N_{0}=\frac{\int_{\mathcal{B}} \Phi(f) \mathrm{d} f}{N_{0}} \leq \frac{C_{1}+C_{2}}{N_{0}}
$$

where equality holds if and only if the bandlimitation step in Eq. (2) is omitted. Further, we define SNR as the product of $C / N_{0}$ and the coherent integration time

$$
\mathrm{SNR}=C / N_{0} \times T_{i}
$$

The Gabor (or root mean square) bandwidth of the meta-signal is given as

$$
\beta=\sqrt{\frac{\int_{\mathcal{B}} \Phi(f) f^{2} \mathrm{~d} f}{\int_{\mathcal{B}} \Phi(f) \mathrm{d} f}}
$$

In the following, we briefly state the CRLB and ZZB from the literature. On the one hand, the well-known CRLB [7] states that the variance of any unbiased ToA estimator $\hat{\tau}$ is bounded below by

$$
\operatorname{Var}(\hat{\tau}) \geq \frac{1}{8 \pi^{2} \mathrm{SNR} \beta^{2}}
$$

While the CRLB is valid for all SNR regions, it can usually only be attained for asymptotically high SNR and therefore is not always a good indicator for the actual performance. [3]. A more accurate bound, which models the estimation variance in all SNR regions more realistically, is given by the $\mathrm{ZZB}$

$$
\operatorname{Var}(\hat{\tau}) \geq \frac{T_{0}^{2}}{6} Q(\sqrt{\mathrm{SNR}})+\frac{1}{2 \beta^{2} \mathrm{SNR}} \Gamma_{\frac{3}{2}}\left(\frac{\mathrm{SNR}}{2}\right)-\frac{4}{3 T_{0} \sqrt{\pi}}\left(\beta^{2} \mathrm{SNR}\right)^{-\frac{3}{2}} \Gamma_{2}\left(\frac{\mathrm{SNR}}{2}\right)
$$

where $Q$ denotes the Q-function and $\Gamma_{a}(z)$ the incomplete gamma function. Unlike the CRLB, the ZZB depends also on the width $T_{0}$ of the search interval for the ToA. For SNR $\rightarrow \infty$, the two lower bounds coincide. For SNR $\rightarrow 0$, the best an unbiased ToA estimator can do is return a uniformly random ToA within $\left[-T_{0} / 2, T_{0} / 2\right]$. Interestingly, the ZZB also characterizes the transition ("waterfall") region, where the performance of optimal estimators begins to deviate from the CRLB. An extensive derivation and study of the ZZB was developed in [8].

\section{Multicorrelator Signal Compression}

The CRLB and ZZB as formulated in the previous section are valid for ToA estimators with knowledge of the full noisy pre-correlation signal $y(t)$. However, some information may be lost in the process of correlating $y(t)$ with a set of time-shifted local replicas of $s(t)$. While this effect is less pronounced for simple signals such as BPSK(1), it is crucial for meta-signals. In the following, we will formulate the CRLB in post-correlation to illustrate this problem. 
We first represent $y(t), t \in\left[0, T_{i}\right]$ by its sampled version. Continuous-time and discrete-time representation are equivalent as long as the sampling rate $1 / T_{s}$ is at least the Nyquist rate. For instance, if the carrier frequency difference is $f_{2}-f_{1}=25.575 \mathrm{MHz}$, then we must sample with at least $1 / T_{s} \geq 2 \times 28.644 \mathrm{MHz}$ to prevent aliasing of the $\operatorname{BOC}(1,1)$ and $\operatorname{BPSK}(1)$ components. The sampled signal is given by

$$
\boldsymbol{y}^{(u)}=\left[\begin{array}{llll}
y(0) & y\left(T_{s}\right) & \ldots & y\left(N T_{s}\right)
\end{array}\right]^{T},
$$

which consists of $N+1=T_{i} / T_{s}$ complex samples. This vector represents the full (uncompressed) signal. On the other hand, the post-correlation (compressed) signal, for $P$ correlators, is

$$
\boldsymbol{y}^{(c)}=\boldsymbol{T}^{H} \boldsymbol{y}^{(u)} \in C^{P}, \quad \mathbf{T}=\left[\begin{array}{llll}
\mathbf{s}\left(\tau_{1}\right) & \mathbf{s}\left(\tau_{2}\right) & \ldots & \mathbf{s}\left(\tau_{P}\right),
\end{array}\right]
$$

where the $P$ columns of the compression matrix $\boldsymbol{T}$ contain the sampled and shifted local replicas

$$
\boldsymbol{s}\left(\tau_{p}\right)=\left[\begin{array}{llll}
s\left(-\tau_{p}\right) & s\left(T_{s}-\tau_{p}\right) & \ldots & s\left(N T_{s}-\tau_{p}\right)
\end{array}\right], \quad p=1, \ldots, P .
$$

The CRLB before correlation is given by

$$
\operatorname{Var}(\hat{\tau}) \geq\left\|\frac{\partial \boldsymbol{s}(\tau)}{\partial \tau}\right\|^{-2}
$$

which is a time-domain representation of (9), while after the correlation it is given by

$$
\operatorname{Var}(\hat{\tau}) \geq\left\|\boldsymbol{T}^{H} \frac{\partial \boldsymbol{s}(\tau)}{\partial \tau}\right\|^{-2}
$$

Therefore, the correlators should be configured in a way that the information loss satisfies

$$
\lambda(\tau)=\frac{\left\|\frac{\partial \boldsymbol{s}(\tau)}{\partial \tau}\right\|^{2}}{\left\|\boldsymbol{T}^{H} \frac{\partial \boldsymbol{s}(\tau)}{\partial \tau}\right\|^{2}} \leq 1+\varepsilon, \quad \forall \tau \in\left[-T_{0} / 2, T_{0} / 2\right]
$$

i.e., that it does not exceed a small tolerable loss with some $\epsilon>0$ for all possible ToAs $\tau$. For example, a tolerable loss would be $10 \log _{10}(1+\varepsilon)=0.1 \mathrm{~dB}$.

For simplicity, we assume that the correlator delays $\tau_{p}, p=1, \ldots, P$, are equally spaced. Moreover, we consider only odd numbers of correlators to ensure that the prompt-correlator is included. Then, suitable combinations of spacing $\Delta_{p}$ and number of correlators $P$ are shown in Fig. 3 for a carrier frequency separation of $f_{2}-f_{1}=25.575 \mathrm{MHz}$. Two things can be observed. First, the less correlators are used, the more accurately should a certain spacing be kept. Second, the number of correlators should not fall short of a certain minimum number (in this example, 13). 


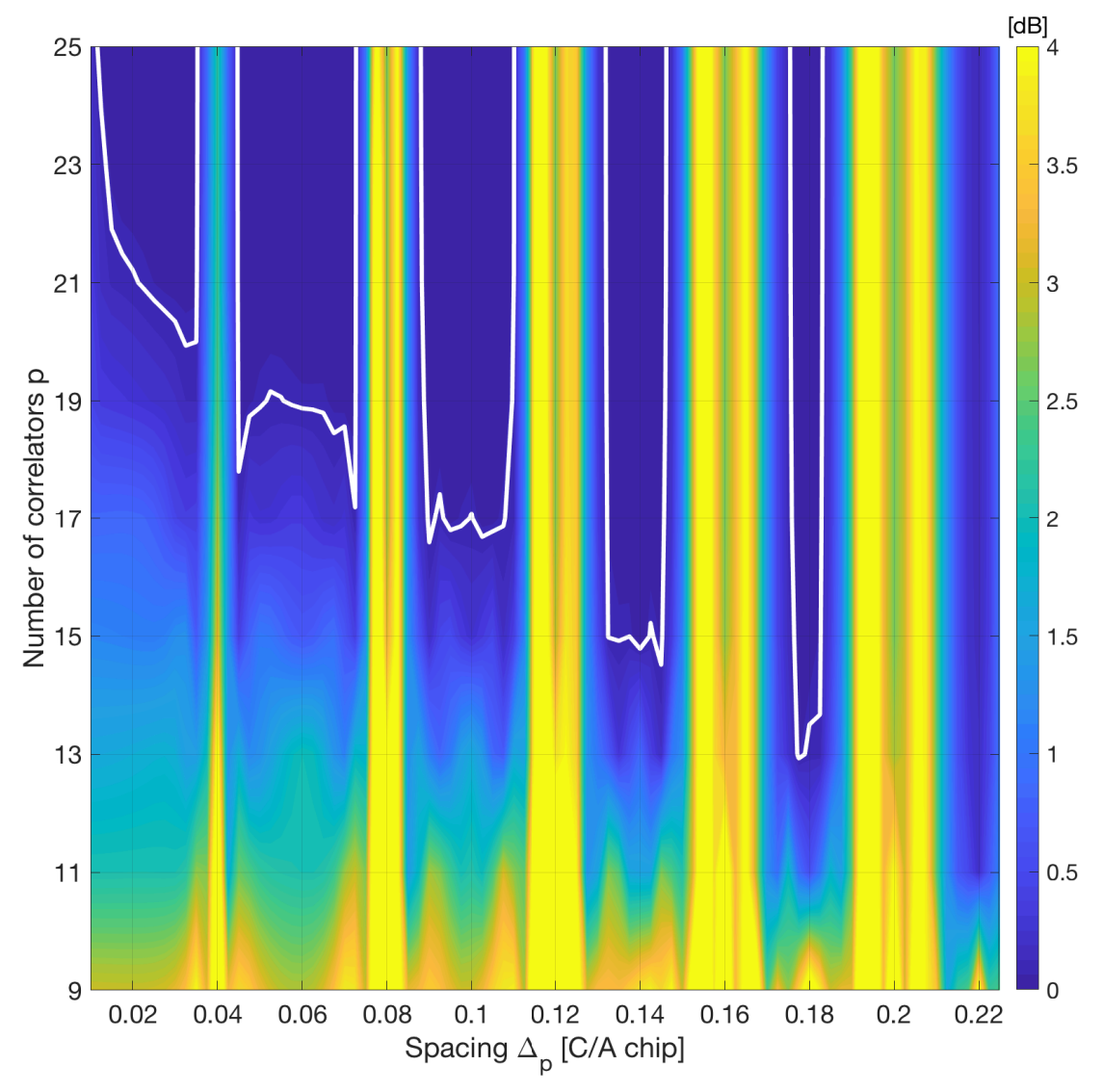

Figure 3: Information loss $10 \log _{10}(\lambda(\tau))$ maximized over $\tau \in\left[-T_{c}, T_{c}\right]$. Those numbers of correlators $P$ and correlator spacings $\Delta_{p}$ which lead to a (quasi-)lossless compression lie within the regions outlined in white. 

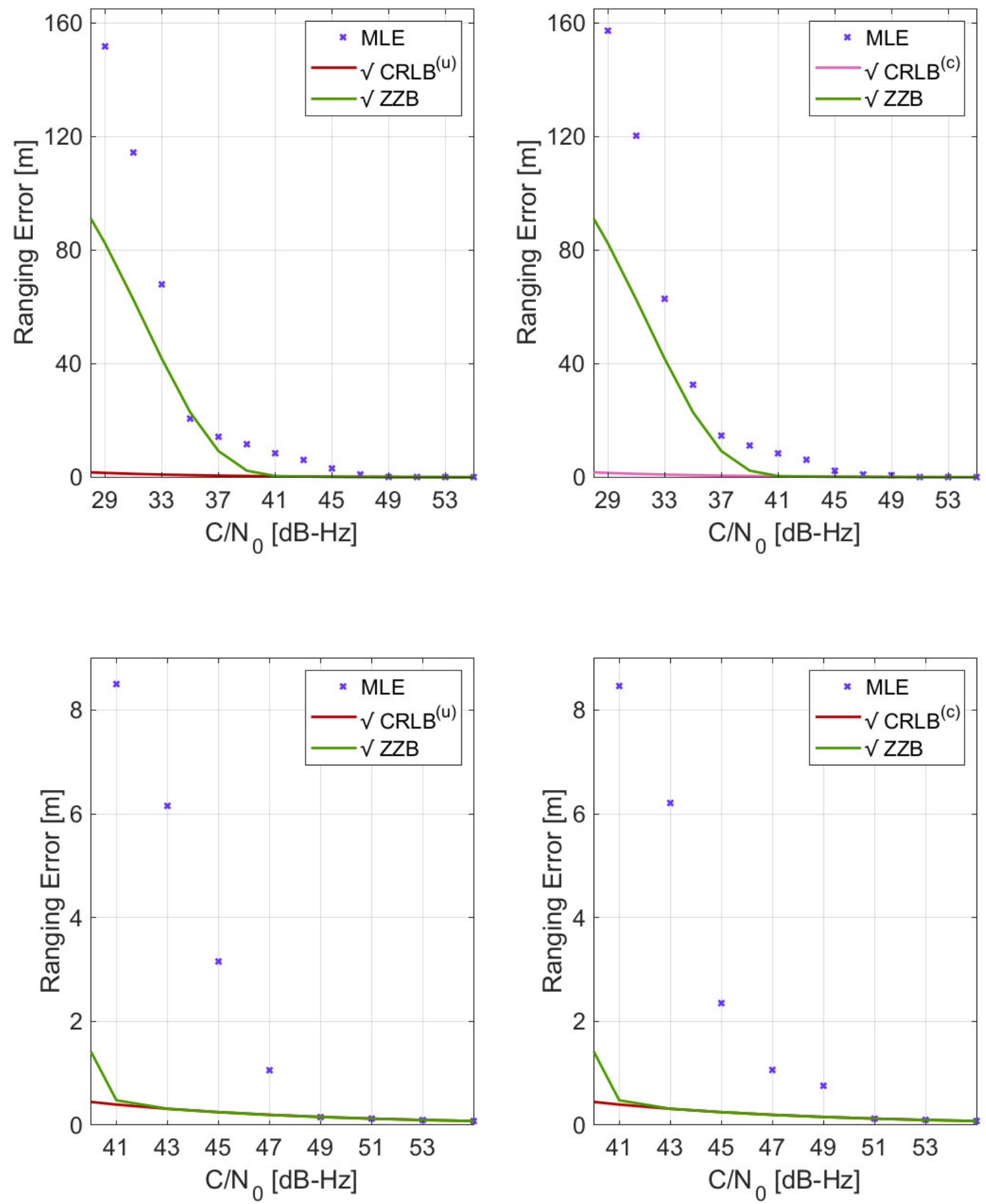

Figure 4: MLE, CRLB and ZZB for the pre-correlation signals (top and bottom left) and post-correlation signals (top and bottom right). $\mathrm{CRLB}^{(u)}$ and $\mathrm{CRLB}^{(c)}$ denote the CRLB for the pre- and post-correlation signals, respectively. 


\section{Simulation Results}

We conducted simulations for a meta-signal with PSD as shown in Fig. 1 and as given in Eq. (2), with carrier frequency difference $f_{2}-f_{1}=25.575 \mathrm{MHz}$. The coherent integration time is always equal to $T_{i}=1 \mathrm{~ms}$. Further, the power of the individual signal components was set to $C_{1}=C_{2}$, while the noise floor is $N_{0}=-204 \mathrm{dBW} / \mathrm{Hz}$. The ToA uncertainty is assumed as $T_{0}=2 T_{c}$. The correlator bank was configured with $p=15$ correlators and a spacing of $\Delta_{p}=0.18 T_{c}$. The results are shown in Fig. 4. We computed the ZZB, as well as the CRLB before and after the compression step. Interestingly, due to the very large sidelobes of the metasignal ACF, the ZZB starts to deviate from the CRLB already when the $C / N_{0}$ goes below 41 $\mathrm{dB}-\mathrm{Hz}$. We also show the performance of an optimal maximum likelihood estimator (MLE), each before and after the multicorrelator compression. (Explicit expressions for the MLE will be provided in the full paper.) It can be observed that the multicorrelator compression loss does not lead to significant performance loss of the MLE, but that asymptotic performance is reached only at around $50 \mathrm{~dB}-\mathrm{Hz}$. However, note that it is possible to operate efficiently in lower $C / N_{0}$ regions if the coherent integration time $T_{i}$ is increased, since SNR (7), not $C / N_{0}$, is decisive for performance.

\section{References}

[1] M. Paonni, J.T. Curran, M Bavaro, J. Fortuny-Guasch, "GNSS Meta-signals: Coherently Composite Processing of Multiple GNSS signals", Proceedings of the 27th International Technical Meeting of the ION Satellite Division, ION GNSS+2014, Tampa, Florida, September 8-12, 2014.

[2] J.-L. Issler, M. Paonni, and B. Eissfeller, "Toward Centimetric Positioning Thanks to L-and S-Band GNSS and to Meta-GNSS Signals", 5th ESA Workshop on Satellite Navigation Technologies and European Workshop on GNSS Signals and Signal Processing (NAVITEC), 2010.

[3] A. Weiss, and E. Weinstein, "Fundamental Limitations in Passive Time Delay Estimation, Part I and II", IEEE Transactions on Acoustics, Speech, and Signal Processing, 1983, vol. 31, no. 2.

[4] P. Fine, and W. Wilson, "Tracking Algorithm for GPS Offset Carrier Signals", Proceedings of the 1999 National Technical Meeting of The Institute of Navigation, San Diego, CA, January 1999, pp. 671-676.

[5] P. Teunissen, and O. Montenbruck, "Springer Handbook of Global Navigation Satellite Systems", Springer Media, 2017.

[6] C. Enneking, "Fisher Information Preserving Compression for GNSS Signals Using SignalMatched Correlators", Technical Note. German Aerospace Center (DLR), 2013.

[7] S. M. Kay, "Fundamentals of Statistical Signal Processing: Vol. 1. Estimation Theory", Prentice Hall, 1993.

[8] K. L. Bell, Y. Steinberg, Y. Ephraim, and H. L. Van Trees, "Extended Ziv-Zakai Lower Bound for Vector Parameter Estimation", IEEE Transactions on Information Theory, 1997, vol. 43, no. 2, pp. 624-637. 